\title{
QED in the clothed-particle representation: a fresh look at positronium properties treatment
}

\author{
Yan A. Kostylenko ${ }^{1,2, \star}$, Adam M. Arslanaliev ${ }^{1,2}$ and Aleksandr V. Shebeko ${ }^{2}$ \\ 1 V.N. Karazin Kharkiv National University, Kharkiv, Ukraine \\ 2 Institute for Theoretical Physics of NSC KIPT, Kharkiv, Ukraine \\ * nlokost@gmail.com
}

October 13, 2019

Proceedings for the 24th edition of European Few Body Conference, Surrey, UK, 2-4 September 2019

\begin{abstract}
We have extended our previous applications of the method of unitary clothing transformations (UCTs) in mesodynamics $[1,2]$ to quantum electrodynamics (QED) $[3,4]$. An analytical expression for the QED Hamiltonian in the clothed-particle representation (CPR) has been derived. Its distinctive feature is the appearance of a new family of the Hermitian and energy independent interaction operators built up in the $e^{2}$-order for the clothed electrons and positrons instead the primary canonical interaction between electromagnetic and electron-positron fields. The problem of describing the bound states in QED in case of the positronium system has been considered. The first correction to the energy of the ground state of the para-positronium and its decay rate to two photons has been calculated by using the new interaction operators.
\end{abstract}

\section{Introduction}

Starting from the primary canonical interaction between electromagnetic and electron-positron fields, the QED Hamiltonian has been expressed through a new family of the Hermitian and energy independent interaction operators built up in the $e^{2}$-order for the clothed electrons and positrons. In this context, we show the QED Hamiltonian $H_{\text {qed }}(\alpha)=H_{F}(\alpha)+V_{\text {qed }}(\alpha)$ in the bare particle representation (see, e.g., the monograph [5]), where $V_{\text {qed }}$ is given by

$$
V_{\text {qed }}=\int d \boldsymbol{x} j_{k}(\boldsymbol{x}) a^{k}(\boldsymbol{x})+V_{\text {Coul }}=V^{(1)}+V_{\text {Coul }},
$$

with the electron-positron current density operator

$$
j_{\mu}(\boldsymbol{x})=e \bar{\psi}(\boldsymbol{x}) \gamma_{\mu} \psi(\boldsymbol{x})
$$

and the Coulomb part

$$
V_{\text {Coul }}=: \frac{1}{2} \int d x \int d y \frac{j^{0}(x) j^{0}(y)}{4 \pi|x-y|} e^{-\lambda|x-y|}: .
$$

In the CPR

$$
H_{\mathrm{qed}}(\alpha)=H_{F}(\alpha)+V_{\mathrm{qed}}(\alpha) \equiv K\left(\alpha_{c}\right)=K_{F}\left(\alpha_{c}\right)+K_{I}\left(\alpha_{c}\right) .
$$


Admittedly the exponential factor with the parameter $\lambda>0$ set to zero at the end of all calculations is introduced to deal with infrared divergences. Here $\alpha_{c}$ denotes the set of all creation and destruction operators for the clothed particles included. Note also that we use the Coulomb gauge (CG), where the photon field $a_{\mu}$ being transverse, has two independent polarizations.

It is proved, that in the $e^{2}$-order the interaction part $K_{I}\left(\alpha_{c}\right)$ is approximated by

$$
\begin{aligned}
K_{I}^{(2)}\left(\alpha_{c}\right)=K_{e^{-} e^{-} \rightarrow e^{-} e^{-}}+K_{e^{+} e^{+} \rightarrow e^{+} e^{+}}+K_{e^{+} e^{-} \rightarrow e^{+} e^{-}} & K_{e^{+} e^{-} \rightarrow \gamma \gamma}+K_{\gamma \gamma \rightarrow e^{+} e^{-}}+ \\
& +K_{e^{-} \gamma \rightarrow e^{-} \gamma}+K_{e^{+} \gamma \rightarrow e^{+} \gamma},
\end{aligned}
$$

where the separate contributions in the r.h.s are responsible for the different physical processes in this system of interacting photons and leptons.

\section{Analytical expressions}

A distinctive feature of our approach is that all expressions in the r.h.s of (5) are obtained simultaneously with mass and vertex renormalizations from the commutator of $V$ from (1) with the generator of the first unitary clothing operator [1]. In particular, we present the interaction operator between clothed electrons and positrons

$$
\begin{gathered}
K_{e^{-} e^{+} \rightarrow e^{-} e^{+}}=\int \frac{d \boldsymbol{p}_{1}^{\prime}}{p_{1}^{\prime 0}} \frac{d \boldsymbol{p}_{2}^{\prime}}{p_{2}^{\prime 0}} \frac{d \boldsymbol{p}_{1}}{p_{1}^{0}} \frac{d \boldsymbol{p}_{2}}{p_{2}^{0}} V_{e^{-} e^{+}}\left(p_{1}^{\prime}, p_{2}^{\prime} ; p_{1}, p_{2}\right) b^{\dagger}\left(p_{1}^{\prime}\right) d^{\dagger}\left(p_{2}^{\prime}\right) b\left(p_{1}\right) d\left(p_{2}\right), \\
V_{e^{-} e^{+}}\left(p_{1}^{\prime}, p_{2}^{\prime} ; p_{1}, p_{2}\right)=\frac{e^{2} m^{2}}{(2 \pi)^{3}} \delta\left(\boldsymbol{p}_{2}^{\prime}+\boldsymbol{p}_{1}^{\prime}-\boldsymbol{p}_{2}-\boldsymbol{p}_{1}\right)\left[v_{S}\left(p_{1}^{\prime}, p_{2}^{\prime} ; p_{1}, p_{2}\right)+v_{A}\left(p_{1}^{\prime}, p_{2}^{\prime} ; p_{1}, p_{2}\right)\right],
\end{gathered}
$$

where $m$ the physical electron (positron) mass, $b(d)$ is the destruction operator for the clothed electron (positron). Henceforth, we omit polarization indices where it does not lead to confusion. In addition, we have introduced the decomposition into the so-called scattering and annihilation contributions $v_{S}$ and $v_{A}$. Each of them has the structure

$$
\begin{gathered}
v_{S / A}=v_{S / A}^{\text {Feynman-like }}+v_{S / A}^{\text {off-energy-shell }}, \\
v_{S}^{\text {Feynman-like }}=-\bar{u}\left(p_{1}^{\prime}\right) \gamma^{\mu} u\left(p_{1}\right) \frac{1}{2}\left\{\frac{1}{\left(p_{1}^{\prime}-p_{1}\right)^{2}}+\frac{1}{\left(p_{2}^{\prime}-p_{2}\right)^{2}}\right\} \bar{v}\left(p_{2}\right) \gamma_{\mu} v\left(p_{2}^{\prime}\right), \\
v_{S}^{\text {off-energy-shell }}=\frac{\left(p_{1}^{\prime}+p_{2}^{\prime}-p_{1}-p_{2}\right)}{\left(p_{1}^{\prime}-p_{1}\right)^{2}+\lambda^{2}} \bar{u}\left(p_{1}^{\prime}\right) \gamma^{0} u\left(p_{1}\right) \frac{1}{2}\left\{\frac{\left(p_{1}^{\prime}-p_{1}\right)}{\left(p_{1}^{\prime}-p_{1}\right)^{2}}+\frac{\left(p_{2}^{\prime}-p_{2}\right)}{\left(p_{2}^{\prime}-p_{2}\right)^{2}}\right\} \bar{v}\left(p_{2}\right) \gamma^{0} v\left(p_{2}^{\prime}\right), \\
v_{A}^{\text {Feynman-like }}=\bar{u}\left(p_{1}^{\prime}\right) \gamma^{\mu} v\left(p_{2}^{\prime}\right) \frac{1}{2}\left\{\frac{1}{\left(p_{1}+p_{2}\right)^{2}}+\frac{1}{\left(p_{1}^{\prime}+p_{2}^{\prime}\right)^{2}}\right\} \bar{v}\left(p_{2}\right) \gamma_{\mu} u\left(p_{1}\right), \\
v_{A}^{\text {off-energy-shell }}=\frac{\left(p_{1}^{\prime}+p_{2}^{\prime}-p_{1}-p_{2}\right)}{\left(p_{1}^{\prime}+p_{2}^{\prime}\right)^{2}+\lambda^{2}} \bar{u}\left(p_{1}^{\prime}\right) \gamma^{0} v\left(p_{2}^{\prime}\right) \frac{1}{2}\left\{\frac{\left(p_{1}^{\prime}+p_{2}^{\prime}\right)}{\left(p_{1}^{\prime}+p_{1}\right)^{2}}-\frac{\left(p_{1}+p_{2}\right)}{\left(p_{1}+p_{2}\right)^{2}}\right\} \bar{v}\left(p_{2}\right) \gamma^{0} u\left(p_{1}\right) .
\end{gathered}
$$

Such a decomposition implies that only the Feynman-like part survives on the energy shell, i.e., on the condition $p_{1}^{\prime 0}+p_{2}^{\prime 0}=p_{1}^{0}+p_{2}^{0}$. Of course, all momenta included are defined on the mass-shell: $p^{2}=p_{0}^{2}-p^{2}=m^{2}$.

Furthermore, we present the operator of the process of the annihilation of clothed electron and positron to two photons

$$
K_{e^{-} e^{+} \rightarrow \gamma \gamma}=\int \frac{d \boldsymbol{k}_{1}}{k_{1}^{0}} \frac{d \boldsymbol{k}_{2}}{k_{2}^{0}} \frac{d \boldsymbol{p}_{1}}{p_{1}^{0}} \frac{d \boldsymbol{p}_{2}}{p_{2}^{0}} V_{e^{-} e^{+} \gamma \gamma}\left(k_{2}, k_{1} ; p_{2}, p_{1}\right) c^{\dagger}\left(k_{2}\right) c^{\dagger}\left(k_{1}\right) b\left(p_{2}\right) d\left(p_{1}\right),
$$


where $c^{\dagger}$ is the creation operator for the clothed photon. Similarly to (8), we separate offenergy-shell part which goes to zero if energy conservation law satisfied

$$
\begin{gathered}
V_{e^{-} e^{+} \gamma \gamma}\left(k_{2}, k_{1} ; p_{2}, p_{1}\right)=\frac{e^{2} m}{2(2 \pi)^{3}} \delta\left(\boldsymbol{p}_{1}+\boldsymbol{p}_{2}-\boldsymbol{k}_{1}-\boldsymbol{k}_{2}\right)\left[v^{\text {Feynman }}+v^{\text {off-energy-shell }}\right], \\
v^{\text {Feynman }}=\frac{\bar{v}\left(p_{1}\right) \phi\left(k_{1}\right) \phi\left(k_{2}\right) u\left(p_{2}\right)}{p_{1}-\not l_{1}+m}, \\
v^{\text {off-energy-shell }}=-\frac{1}{2}\left[\frac{\bar{v}\left(p_{1}\right) \phi\left(k_{1}\right) \phi\left(k_{2}\right) u\left(p_{2}\right)}{\not_{1}-\not l_{1}+m}+\frac{\bar{v}\left(p_{1}\right) \phi\left(k_{2}\right) \phi\left(k_{1}\right) u\left(p_{2}\right)}{\not_{2}-\not k_{1}-m}\right] .
\end{gathered}
$$

\section{Correction to the positronium ground state energy}

The problem of describing the bound states in QED in case of the positronium (Ps) has been considered by using the new interaction (6). Positronium consisting of an electron and a positron is the simplest bound system in QED. Its ground state (g.s.) has two possible configurations with total spin values $S=0,1$. The singlet (triplet) lowest-energy state with $S=0$ $(\mathrm{S}=1)$ is known as the para-positronium (ortho-positronium). For this exposition, we will restrict ourselves to the consideration of the para-positronium (p-Ps) system.

As noted in [6], the Fock subspace of all the clothed states can be divided into several sectors (two electrons sector, photon-electron sector, etc.) such that $K^{(2)}\left(\alpha_{c}\right)$ leaves each of them to be invariant, i.e., for any state vector $|\Phi\rangle$ of such sector $K^{(2)}\left(\alpha_{c}\right)|\Phi\rangle$ belongs to the same sector. Here we make an assumption that the Ps state belongs only to electron-positron sector. The corresponding g.s., being the $H$ eigenvector, viz.,

$$
H|\mathrm{p}-\operatorname{Ps}(\boldsymbol{P})\rangle=E|\mathrm{p}-\operatorname{Ps}(\boldsymbol{P})\rangle,
$$

can be represented as

$$
|\boldsymbol{P} ; \mathrm{p}-\mathrm{Ps}\rangle=\int \frac{d \boldsymbol{p}_{1}}{p_{1}^{0}} \frac{d \boldsymbol{p}_{2}}{p_{2}^{0}} \Psi_{00}\left(\boldsymbol{P} ; \boldsymbol{p}_{1}, \boldsymbol{p}_{2}\right) b^{\dagger}\left(p_{1}\right) d^{\dagger}\left(p_{2}\right)|\Omega\rangle .
$$

In the p-Ps rest system or center mass system (c.m.s.) the eigenvalue equation has the form

$$
2 p_{0} \Psi_{00}(\boldsymbol{p})+\int \frac{d \boldsymbol{p}^{\prime}}{p_{0} p_{0}^{\prime}} \bar{V}\left(p^{\prime}, p\right) \Psi_{00}\left(\boldsymbol{p}^{\prime}\right)=m_{\mathrm{p}-\mathrm{Ps}} \Psi_{00}(\boldsymbol{p}),
$$

where $m_{\mathrm{p}-\mathrm{Ps}}=m_{e^{-}}+m_{e^{+}}+\varepsilon_{\mathrm{p} \text {-Ps }}$ the para-positronium mass and $\varepsilon_{\mathrm{p}-\mathrm{Ps}}$ its binding energy, $\Psi_{00}(\boldsymbol{p}) \equiv \Psi_{00}(\boldsymbol{P}=0 ; \boldsymbol{p},-\boldsymbol{p})$ (since we work in c.m.s.) and $\bar{V}\left(p^{\prime}, p\right)$ gets out from (6) in c.m.s. $\left(\boldsymbol{p} \equiv \boldsymbol{p}_{1}=-\boldsymbol{p}_{2}, \boldsymbol{p}^{\prime} \equiv \boldsymbol{p}_{1}^{\prime}=-\boldsymbol{p}_{2}^{\prime}\right)$

$$
\bar{V}\left(p_{1}^{\prime}, p_{2}^{\prime}, p_{1}, p_{2}\right)=\left\langle\Omega\left|d\left(p_{2}^{\prime}\right) b\left(p_{1}^{\prime}\right) K_{e^{-} e^{+} \rightarrow e^{-} e^{+}} b^{\dagger}\left(p_{1}\right) d^{\dagger}\left(p_{2}\right)\right| \Omega\right\rangle .
$$

In the non-relativistic limit $\left(p_{0}=p_{0}^{\prime}=m\right)$ the eigenvalue equation reduces to the ordinary Schrödinger equation for the Coulomb potential in momentum space. Therefore we come to the well-known Coulomb problem with the g.s. energy $\varepsilon_{\text {g.s. }} \approx-6.8 \mathrm{eV}$. By considering the difference between $\bar{V}\left(p^{\prime}, p\right)$ and the Coulomb potential as a perturbation (it is not evident) and using the non-perturbative wave function of the ground state

$$
\Psi_{00}(p)=\frac{2}{\pi} \frac{\sqrt{2 a^{3}}}{\left(1+a^{2} p^{2}\right)^{2}}
$$


from Appendix C in [7] we have computed the energy shift

$$
\Delta \varepsilon=-4.7325 \cdot 10^{-4} \mathrm{eV} .
$$

This value surprisingly coincides with those estimations given in [7] (see formula (1.1) therein). In order to verify such a coincidence beyond the perturbation theory, we are addressing the partial wave decomposition of the positronium eigenvectors that has been successful when finding the $u$ and $w$ components of the deuteron wave function (WF) [8].

\section{The partial eigenvalue equation for para-positronium}

In this context, we derive the partial eigenvalue equation for the para-positronium WFs that belong to the total angular momentum J, viz.,

$$
2 p_{0} \Psi^{J}(p)+\int_{0}^{\infty} \frac{p^{\prime 2} d p^{\prime}}{p_{0} p_{0}^{\prime}} \Psi^{J}\left(p^{\prime}\right) \bar{V}^{J}\left(p, p^{\prime}\right)=m_{\mathrm{p}-\mathrm{Ps}} \Psi^{J}(p) .
$$

Here $\bar{V}^{J}\left(p, p^{\prime}\right)$ is the partial electron-positron quasipotential derived in the momentum representation from the new $e^{-} e^{+}$-interaction operator. In turn, we have

$$
\left.\bar{V}^{J}\left(p, p^{\prime}\right)=\bar{v}^{J} \text { (Feynman-like }\right)+\bar{v}^{J} \text { (off-energy-shell). }
$$

Such a separation implies that only the Feynman-like part survives on the energy shell, where $p_{0}^{\prime}=\sqrt{p^{\prime 2}+m^{2}}=p_{0}=\sqrt{p^{2}+m^{2}}$. The task of solving the eigenvalue equation and obtaining the corresponding positronium states in the CPR is underway (see Appendix C in [9]).

\section{Positronium decay rates}

The positronium decay to two photons has considered. The corresponding decay rate is given by (see formula (9.337) in [10])

$$
\Gamma=\sum_{\sigma_{1} \sigma_{2}} \int \frac{d \boldsymbol{k}_{1}}{k_{1}^{0}} \int \frac{d \boldsymbol{k}_{2}}{k_{2}^{0}} \pi \delta\left(k_{1}^{0}+k_{2}^{0}-E_{\mathrm{Ps}}\right) \delta\left(\boldsymbol{k}_{1}+\boldsymbol{k}_{2}-\boldsymbol{P}\right)\left|T_{f i}\right|^{2},
$$

where the T-matrix element $T_{f i}=\left\langle\Omega\left|c\left(k_{1} \sigma_{1}\right) c\left(k_{2} \sigma_{2}\right) T\right| P s\right\rangle$ from the initial Ps ground state to the final state of the two photons, respectively, with the momenta $k_{1}=\left(k_{1}^{0}, \boldsymbol{k}_{1}\right)$ and $k_{2}=\left(k_{2}^{0}, \boldsymbol{k}_{2}\right)$ and their polarizations $\sigma_{1}, \sigma_{2}$. In this connection, we note an equivalence theorem proved in [11], that allows us to use a recipe for calculating the S-matrix (T-matrix) in the CPR.

In the rest frame of positronium $(\boldsymbol{P}=0)$ one can do integration with both $\delta$-functions

$$
\Gamma=\frac{\pi}{2} \sum_{\sigma_{1} \sigma_{2}} \int d \hat{\boldsymbol{k}}_{1}\left|T_{f i}\right|^{2}
$$

where $\hat{\boldsymbol{k}}_{1}$ is the unit vector along vector $\boldsymbol{k}_{1}$.

The positronium state-vector with spin $S$ can be represented as

$$
|P s(S)\rangle=\sum_{M_{S}} \int \frac{d \boldsymbol{p}}{p^{0}} \Psi_{S M_{S}}(\boldsymbol{p})\left|p S M_{S}\right\rangle,
$$


where $\left|p S M_{S}\right\rangle=\sum_{\mu_{1} \mu_{2}}\left(\frac{1}{2} \mu_{1} \frac{1}{2} \mu_{2} \mid S M_{S}\right) b^{\dagger}\left(p_{-} \mu_{2}\right) d^{\dagger}\left(p \mu_{1}\right)|\Omega\rangle$ the electron-positron state-vector in the total spin representation and $p_{-}=\left(p_{0},-p\right)$. In the Born approximation where $T \approx K_{e^{-}} e^{+} \rightarrow \gamma \gamma$ one has

$$
\begin{aligned}
& \Gamma=\frac{\pi}{2} \sum_{\sigma_{1} \sigma_{2}} \int d \hat{\boldsymbol{k}}\left|\left\langle k \sigma_{1} \sigma_{2}\left|K_{e^{-} e^{+} \rightarrow \gamma \gamma}\right| \mathrm{p}-\mathrm{Ps}\right\rangle\right|^{2}, \\
& \left\langle k \sigma_{1} \sigma_{2}\left|K_{e^{-} e^{+} \rightarrow \gamma \gamma}\right| \mathrm{p}-\mathrm{Ps}\right\rangle=\int \frac{d \boldsymbol{p}}{p_{0}} \Psi_{00}(\boldsymbol{p})\left\langle k \sigma_{1} \sigma_{2}\left|K_{e^{-} e^{+} \rightarrow \gamma \gamma}\right| p 00\right\rangle,
\end{aligned}
$$

where $\left\langle k \sigma_{1} \sigma_{2}\right| \equiv\langle\Omega| c\left(k \sigma_{1}\right) c\left(k_{-} \sigma_{2}\right)$. Here we use the g.s. Coulomb wave function as an approximation.

The interaction (9) in an arbitrary frame has the form

$$
\begin{gathered}
\left\langle\Omega\left|c\left(k_{1} \sigma_{1}\right) c\left(k_{2} \sigma_{2}\right) K_{e^{-} e^{+} \rightarrow \gamma \gamma} b^{\dagger}\left(p_{2} \mu_{2}\right) d^{\dagger}\left(p_{1} \mu_{1}\right)\right| \Omega\right\rangle=\frac{\alpha m}{4 \pi^{2}} \bar{v}_{e^{-} e^{+} \gamma \gamma}, \\
\bar{v}_{e^{-} e^{+} \gamma \gamma}=\bar{v}\left(p_{1} \mu_{1}\right) \phi\left(k_{1} \sigma_{1}\right) \frac{1}{2}\left\{\frac{1}{\not p_{2}-\not l_{2}-m}-\frac{1}{p_{1}-\not l_{1}+m}\right\} \phi\left(k_{2} \sigma_{2}\right) u\left(p_{2} \mu_{2}\right)+ \\
+\left(k_{1}, \sigma_{1} \leftrightarrow k_{2}, \sigma_{2}\right),
\end{gathered}
$$

whence in the static limit $\left(\boldsymbol{p}_{1,2}=0\right)$ we arrive to the well known Pirenne-Wheeler result with $\Gamma=\frac{1}{2} \alpha^{5} m \approx 8.0325 \cdot 10^{9} \sec ^{-1}[12,13]$. After this by using the CG we get

$$
\Gamma=\frac{128}{\pi^{2}} m \alpha^{7} \mathrm{I}(\alpha)^{2}
$$

with the integral

$$
\mathrm{I}(\alpha)=\frac{1}{8} \int_{0}^{\infty} d u \frac{u \sinh u}{\left(\alpha^{2} / 4+\sinh ^{2} u\right)^{2}}=26.7535 .
$$

Now we get the decay rate of para-positronium into two photons $\Gamma=7.9411 \cdot 10^{9} \mathrm{sec}^{-1}$. The experimental result [14] for this value $7.9909 \pm 0.0017 \cdot 10^{9} \mathrm{sec}^{-1}$.

\section{Conclusion}

We have shown that the UCT method can be successfully applied to the treatment of the bound states in QED. Our consideration gives one more application of a well-forgotten concept on the clothed particles in quantum field theory, put forward by Greenberg and Schweber [15]. We have seen that our approach leads to new hermitian and energy independent interactions between clothed particles including the off-energy-shell and recoil effects (the latter in all orders of the $v^{2} / c^{2}$ - expansion).

\section{Acknowledgements}

Two of us, Y.K. and A.A. are very grateful to the Organizing Committee of the 24th European Conference on Few-Body Problems in Physics and the International Relations Office of V. N. Karazin Kharkiv National University for the financial assistance, of our participation in this conference. 


\section{References}

[1] A. Shebeko and M. Shirokov, Clothing procedure in relativistic quantum field theory and its applications to description of electromagnetic interactions with nuclei (bound systems), Progr. Part. Nucl. Phys. 44, 75 (2000), doi:10.1016/S0146-6410(00)00060-0.

[2] V. Korda, L. Canton and A. Shebeko, Relativistic interactions for the meson-two-nucleon system in the clothed-particle unitary representation, Ann. Phys. 322, 736 (2007), doi:10.1016/j.aop.2006.07.010.

[3] A. Shebeko, Clothed particles in mesodynamics, quantum electrodynamics and other field models, PoS Baldin ISHEPP XXII 225, 027 (2015), doi:10.22323/1.225.0027.

[4] A. Arslanaliev, The method of unitary clothing transformations: application in quantum electrodynamics, Master's thesis, V.N. Karazin National University, Kharkov, Ukraine (2017).

[5] S. Weinberg, The Quantum Theory of Fields, vol. 1, Cambridge University Press (1995), doi:10.1017/СBO9781139644167.

[6] A. Shebeko and M. Shirokov, Unitary transformations in quantum field theory and bound states, Phys. Part. Nucl. 32, 15 (2001), https://arxiv.org/abs/nucl-th/0102037.

[7] C. Smith, Bound State Description in Quantum Electrodynamics and Chromodynamics, Ph.D. thesis, Louvain-la-Neuve (2002).

[8] A. Shebeko, Ch. 1 In Advances in quantum field theory, ed. S. Ketov, INTECH, 3 (2012), doi:10.5772/37563.

[9] I. Dubovyk and A. Shebeko, The method of unitary clothing transformations in the theory of nucleon-nucleon scattering, Few-Body Syst. 48, 109 (2010), doi:10.1007/s00601-0100097-5.

[10] H. Kleinert, Particles and Quantum Fields, Freie Universität Berlin (2016), doi:10.1142/9915.

[11] A. Shebeko, The s-matrix in the method of unitary clothing transformations, Nucl. Phys. A 737, 252 (2004).

[12] J. Wheeler, Polyelectrons, Ann. N. Y. Acad. Sci. 48, 219 (1946), doi:10.1111/j.17496632.1946.tb31764.x.

[13] J. Pirenne, The proper field and the interaction of Dirac particles, Arch. Sci. Phys. Nat. 28, 233 (1946).

[14] A. Al-Ramadhan and D. Gidley, New precision measurement of the decay rate of singlet positronium, Phys. Rev. Lett. 72, 1632 (1994), doi:10.1103/PhysRevLett.72.1632.

[15] O. Greenberg and S. Schweber, Clothed particle operators in simple models of quantum field theory, Nuovo Cim. 8, 378 (1958), doi:10.1007/BF02828746. 